\title{
COMPARATIVE STUDY BETWEEN AUTOLOGOUS BLOOD AND PLATELET RICH PLASMA IN TREATMENT OF RECURRENT TEMPOROMANDIBULAR JOINT DISLOCATION
}

\author{
Menatallah M Yasso ${ }^{1} B D S$, Magued H Fahmy . ${ }^{2} P h D$, Nevine S Mohamad. ${ }^{3} P h D$
}

\begin{abstract}
INTRODUCTION: Temporomandibular joint (TMJ) dislocation is an excessive forward movement of the condyle beyond the articular eminence with complete separation of the articular surfaces and fixation in that position.

OBJECTIVES: the aim of the study was to compare clinically between the effect of autologous blood injection and platelet rich plasma injection in treatment of chronic recurrent temporomandibular joint dislocation through evaluation of mandibular range of motion.

MATERIALS AND METHODS: Twenty patients with bilateral chronic recurrent condylar dislocation were included in this study. In group I 3mL autologous blood was injected. For group II 3ml of concentrated platelet rich plasma (PRP) was injected.

Preoperative and postoperative assessment included a thorough history and physical examination to determine mandibular range of motion.

RESULTS: Mandibular range of motion was significantly reduced post operatively in both groups, however on comparing the two groups there was statistically insignificant difference in mandibular range of motion.

CONCLUSIONS: Both autologous blood and PRP injections are simple, low cost, and minimally invasive approaches which can be easily and safely injected. Both Autologous blood injection and PRP significantly decrease mandibular range of motion to normal range in patients with chronic recurrent TMJ dislocation.
\end{abstract}

KEYWORDS: Temporomandibular joint, dislocation, platelet rich plasma, blood.

1- Bachelor of Faculty of Dentistry 2011, Alexandria University.

2-Professor of Oral and Maxillofacial Surgery, Faculty of Dentistry, Alexandria University.

3-Professor of Oral and Maxillofacial Surgery, Faculty of Dentistry, Alexandria University.

\section{INTRODUCTION}

The temporomandibular joint (TMJ) is a complex joint with unique characteristics and functions. It consists of fibrocartilaginous disk positioned between the mandibular fossa and the mandibular condyle separating the joint into an upper compartment, which is involved in translational movement, and a lower compartment, which is involved in rotational movement (1)

TMJ hypermobility is divided into two groups: subluxation and dislocation (2). TMJ dislocation occurs when the condyle moves beyond the anterior surface of the articular eminence, stays locked and is unable to reduce itself back to the original position in the glenoid fossa. This condition can occur unilaterally or bilaterally (3). TMJ dislocation may be reducible when it returns spontaneously to the glenoid cavity, or irreducible when one or two condyles remain dislocated (4). In this position, the mouth remains opened due to the action of the elevator muscles with or without lateral deviation, depending on whether the dislocation is unilateral or bilateral (5).

Acute dislocation of the TMJ is usually managed by manually pushing the mandible downward and backward to relocate the condyles in the glenoid fossa. TMJ dislocation can happen during routine daily activities such as speaking, yawning, vomiting, laughing and mastication or may occur during procedures such as laryngoscopy (for intubation), endoscopic procedures and dental treatments (6).

When this problem continues to repeat several times, it is described as chronic recurrent TMJ dislocation. The pathogenesis of chronic recurrent TMJ dislocation is attributed to a combination of factors including laxity of the TMJ ligaments, weakness of the TMJ capsule, an unusual eminence size, muscle hyperactivity or spasms, trauma, and abnormal chewing movements that do not allow the condyle to translate back (7). Complications of recurrent TMJ dislocation include injury to the disc, the capsule and the ligaments leading to progressive TMJ internal derangement and pain (8). Several surgical and non-surgical techniques have been introduced for treating patients with chronic recurrent TMJ dislocation (9).

The non-surgical (conservative) approaches include restriction of mandibular movement (plus muscle relaxants prescription and soft diet), application of local anesthetics, injection of botulinum toxin to the muscles of mastication (10) and injection of sclerosing agents (11). When the nonsurgical modalities are not successful in treating patients with recurrent TMJ dislocation, surgical approaches are considered (11).

The surgical (invasive) approaches include capsular plication (12), reduction or augmentation of articular eminence (13)' temporalis tendon scarification (14), lateral pterygoid myotomy (15) and condylectomy (16).

Unfortunately, significant number of patients failed to respond to basic treatment (17). Even invasive treatment for these patients is often of limited success. Injections of the joints with sclerosing agents, hyaluronic acid, botulinum toxin A ,blood and platelet rich plasma in case of TMJ dislocation have proven to be valuable means of effective local treatment (18).

Autologous blood injection into the TMJ for the treatment of hypermobility is a simple yet effective technique that can be performed on an outpatient basis under local anesthesia (19). It is a minimally invasive modality that is safe ,cost effective, with minimal post-operative complications and without foreign body reactions, moreover it induces fibrosis, 
formation of adhesions and scarring in the joint stopping the recurrent dislocation of the TMJ (20).

Platelet rich plasma (PRP) is a production of autologous blood containing 3-8 folds the concentration of platelets which are obtained by concentrating the blood by gradient density centrifugation (21). PRP is therefore a natural concentrate of growth factors which when injected reduce inflammation, provide pain relief, improve function and stimulate possible cartilage regeneration at the site of injury. Platelets play an important role in coagulation, hemostasis, have analgesic properties by releasing protease activated receptor 4 peptides and once activated platelets secrete growth factors such as (platelet derived growth factor ,transforming growth factor beta, vascular endothelial growth factor ) which are involved in the healing process (22).

In this study comparison between autologous blood and PRP intraarticular injection in the treatment of chronic recurrent TMJ dislocation was assessed.

\section{MATERIALS AND METHODS}

Twenty patients with confirmed diagnosis of chronic recurrent TMJ dislocation were selected in this study from those presented at the clinic of Oral and Maxillofacial Surgery department, Faculty of Dentistry, Alexandria University. Appropriate ethical clearance was obtained from the ethical committee of the faculty of dentistry Alexandria University.

Patients were divided into two groups

Group I: 10 patients were treated by injection of autologous blood; $2 \mathrm{ml}$ into the superior joint cavity and $1 \mathrm{ml}$ to the pericapsular tissues of the TMJ.

Group II: 10 patients were treated by injection of platelet rich plasma; $2 \mathrm{ml}$ into the superior joint cavity and $1 \mathrm{ml}$ to the pericapsular tissues of the TMJ.

\section{Criteria for patient selection}

\section{Inclusion criteria}

Patients diagnosed with chronic recurrent TMJ dislocation clinically and radiographically by Cone beam computed tomography (CBCT) scan, patients diagnosed with bilateral dislocation, dislocation episode ranged from one time to several times per week and patients were able to self-reduce the dislocation.

\section{Exclusion criteria}

Those suffering from systemic disease (platelet function disorders, fibrinogen deficiency), those with previous TMJ surgery and those suffering from joint fractures.

Informed consents were submitted by the patients after informing them thoroughly about the operation and the risks of the operation.

The clinical procedures include preoperative, operative and post-operative phases.

\section{I- The Preoperative Phase}

The diagnosis of cases of chronic recurrent bilateral dislocation of TMJ was determined by a questionnaire, clinical examination and radiographic examination through CBCT. All patients were informed preoperatively about the procedure.

\section{a. Patient questionnaire}

All details were recorded in a questionnaire by the examiner including:

Personal data:patient's name, age, address, telephone number, gender, marital status, and occupation.

Past history: chronic systemic diseases, routine use of medications, history of frequent headache or chronic ear pain, history of serious accidents or injuries to the head, oral habits as grinding or clenching, occurrence of stressful life situations especially around onset of TMJ symptoms.

Chief complaint: history of present illness was recorded including: nature of the problem described in the patient's words, time of onset of symptoms, which side hurts (right or left or both joints), when is the pain worse, presence of joint sounds, history of locking, and previous treatments.

Pain level was determined by patient's self-assessment using a visual analog scale (VASI) from zero to 10 ("0" is pain free and “ 10 ” is severe intolerable pain) (9).

\section{II-The operative phase}

The TMJ was palpated during mandibular movements to locate the condyle. The ear and preauricular skin over the TMJ were cleaned with Betadine solution and the area was isolated with sterile towels. Two points were marked over the auricular fossa and eminence, $1 \mathrm{~cm}$ in front of the tragus along the lateral canthal tragus line and $2 \mathrm{~mm}$ below the tragus. The subcutaneous tissues lateral to the joint were infiltrated with a local anaesthetic solution Mupivacaine (Mepevacaine HCL 2\%, Alexandria Co. for pharmaceuticals Alexandria, Egypt), then the auriculotemporal nerve was blocked by injection of the anaesthetic solution posterior to the condylar neck.

\section{Group I: Autologous blood injection:(figure 1,2)}

For group I, $3 \mathrm{ml}$ of autologous blood was withdrawn from the patients' median cubital vein. A small volume of the patient's blood ( $3 \mathrm{ml})$ was obtained and injected directly, 2 $\mathrm{ml}$ into the superior joint space and $1 \mathrm{ml}$ into the pericapsular tissues.

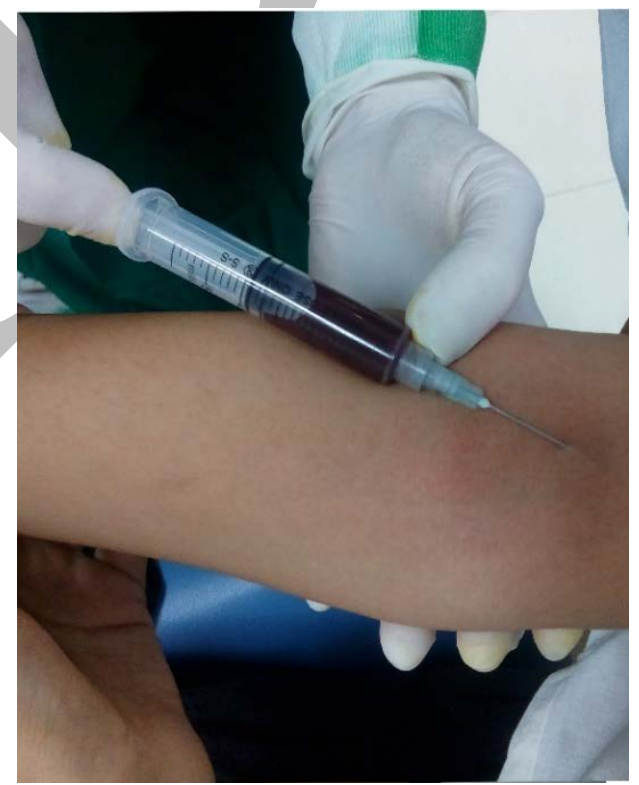

Figure 1: Blood withdrawal procedure. 


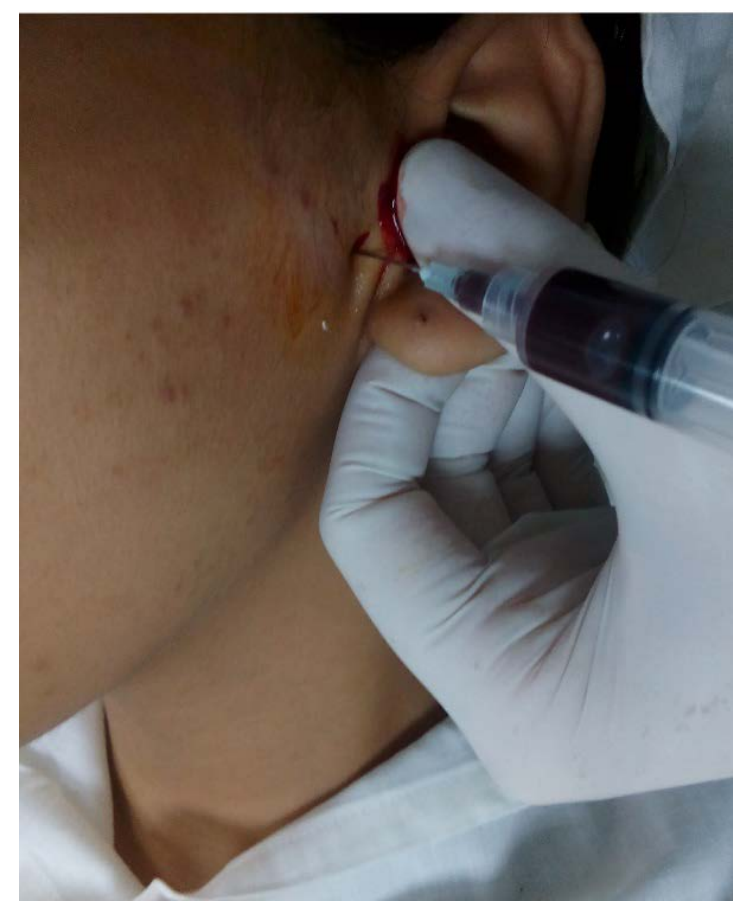

Figure 2: Autologous blood injection into the TMJ.

\section{Group II: PRP injection (figure 3,4)}

In group II PRP was prepared by collecting $10 \mathrm{ml}$ blood from median cubital vein of the patient in a glass centrifuge tube with sodium citrate (3.52) as an anticoagulant under sterile aseptic condition. $10 \mathrm{ml}$ collected blood was mixed with the citrate using rotational movements; an even number of tubes was placed in a centrifuge machine at 3200 rpm for 12 minutes. After separation of the erythrocytes mass, the platelet rich plasma was carefully aspirated into a separate syringe. $2 \mathrm{ml}$ PRP was injected into the upper joint space and $1 \mathrm{ml}$ was injected into the pericapsular tissues; the point of injection was located along the canthotragal line, $10 \mathrm{~mm}$ from the middle of the tragus and $2 \mathrm{~mm}$ below the line. After injection, the patient was asked to open and close the mouth several times for a minute to ensure equal distribution of PRP before it converts into gel (21).

\section{III-The Post-operative phase}

An elastic head bandage was applied for one week and all patients were instructed to restrict their mouth opening and to eat only soft foods for 7 days. Antibiotic Augmentin 1gm tablets (each tablet contains $875 \mathrm{mg}$ amoxicillin potassium and 125 clavulanate ,GSK glaxosmithkline ) given twice daily for 5 days. Analgesic Cataflam tablets $50 \mathrm{mg}$ (Diclofenac potassium, NOVARTS ) given 3 times daily for 3 days. Mandibular Exercises 4 times daily after first week. IV-Post- operative evaluation parameters

Clinical examination was done after 2 weeks, one month and 3 months postoperatively. Clinical evaluation of mandibular range of motion was carried out. For measurement of Maximal interincisal opening (MIO), a caliber was used and the patients were asked to open their mouth as wide as possible then the vertical distance between the incisal edges of upper and lower incisors was measured. For protrusive movement measurement, the patients were asked to protrude their mandible as much as they can and the horizontal distance between the incisal edges of upper and lower incisors were measured.

\section{Statistical method}

Paired t-test was used to test the significant difference in maximal interincisal opening and protrusive movement between the two studied group.
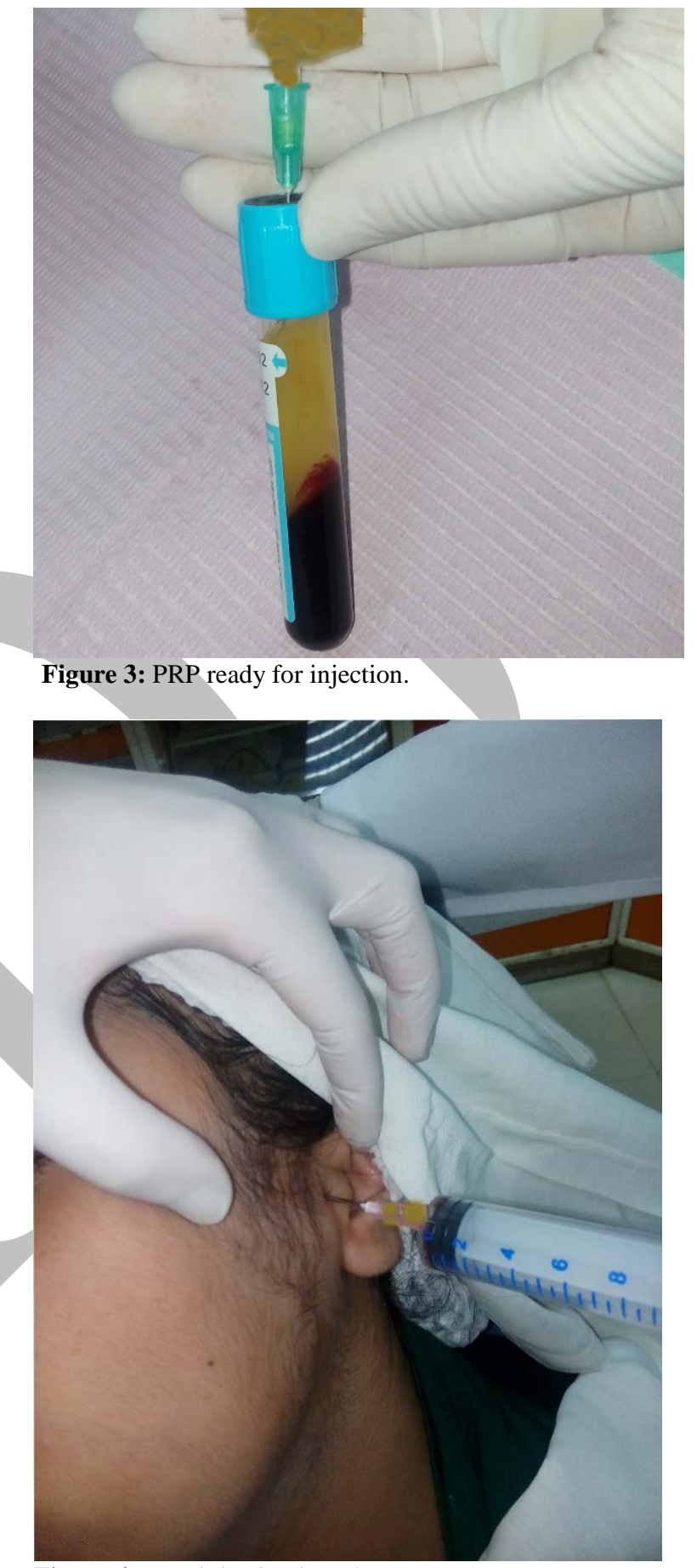

Figure 4: PRP injection into the TMJ.

\section{RESULTS}

The present study has been conducted on twenty patients selected from those attending the outpatient clinic of the Oral and maxillofacial Surgery Department, Faculty of Dentistry, Alexandria University.

All patients were evaluated by clinical diagnosis pre and post-operatively to detect any improvement in clinical signs and symptoms. 


\section{A) Maximal interincisal opening (MIO) (table 1) Preoperatively}

The mean MIO for group I was $44.5+1.3 \mathrm{~mm}$ while the mean MIO for group II was $44.3+3.1 \mathrm{~mm}$.

There was no statistically significant difference between the two groups regarding the maximal interincisal opening preoperatively ( $p$-value $=0.45$ ).

\section{After 2 weeks:}

The mean MIO for group I was 35.8+ $2.3 \mathrm{~mm}$ while the mean MIO for group II was $38.1+1.1 \mathrm{~mm}$. The decrease in maximal interincisal opening from preoperative to 2 weeks postoperative was found to be statistically significant in group I (p-value=0.0001) and also in group II ( p-value $=0.0001)$.

\section{After 4 weeks:}

The mean MIO for group I was $34.1+1.9 \mathrm{~mm}$ while the mean MIO for group II was $33.5+1.2 \mathrm{~mm}$. The decrease in maximal interincisal opening from preoperative to 1 month postoperative was found to be statistically significant in group I ( $p$-value $=0.0001)$ and also in group II (p-value $=0.0001)$.

After 3 months:The mean MIO for group I was 32.1+ 1.1 $\mathrm{mm}$ while the mean MIO for group II was $32.8+1.3 \mathrm{~mm}$. The decrease in maximal interincisal opening from preoperative to 3 months postoperative was found to be statistically significant in group I (p-value $=0.0001)$ and also in group II (p-value $=0.0001)$. Regarding maximal interincisal opening, the difference between the two groups throughout the whole follow-up period was found to be statistically insignificant ( $\mathrm{p}<0.05)$.

Table (1): Comparison between the two studied groups according to MIO

\begin{tabular}{|c|c|c|c|c|}
\hline & Pre-operative & 2 weeks & 1month & 3 months \\
\hline Group I & & & & \\
\hline Min. - Max. & $42-46$ & $33-39$ & $30-35$ & $31-34$ \\
\hline Mean \pm SD & $44.5 \pm 1.3$ & & $34.1 \pm 1.9$ & $32.1 \pm 1.1$ \\
\hline Median & 43 & 35 & 33 & 32 \\
\hline $\mathbf{p}_{1}$ & & $0.0001^{*}$ & $0.0001^{*}$ & $0.0001^{*}$ \\
\hline Group II & & & & \\
\hline Min. - Max. & $42-48$ & $33-39$ & $30-36$ & $31-35$ \\
\hline Mean \pm SD & $44.3 \pm 3.1$ & $\begin{array}{c}30.1 \pm \\
1.1\end{array}$ & $33.5 \pm 1.2$ & $32.8 \pm 1.3$ \\
\hline Median & 43 & 35 & 33 & 32 \\
\hline $\mathbf{p}_{1}$ & & $0.0001^{*}$ & $0.0001^{*}$ & $0.0001^{*}$ \\
\hline $\mathbf{P}_{2}$ & 0.45 & 0.29 & 0.3 & 0.19 \\
\hline
\end{tabular}

$\mathrm{p}_{1}$ : $\mathrm{p}$ value for paired t-test between pre-

operative and each other periods

$\mathrm{p}_{2}$ : $\mathrm{p}$ value for paired t-test between

the two groups of the same period

*: Statistically significant at $\mathrm{p} \leq 0.05$.

\section{B) Protrusive movement (table 2)}

\section{Preoperatively:}

The mean protrusive movement for group I was $12.3+2 \mathrm{~mm}$ while the mean protrusive movement for group II was 12.1 $+1.4 \mathrm{~mm}$. There was no statistically significant difference between the 2 groups. Regarding the protrusive movement preoperatively ( $\mathrm{p}$-value $=0.43$ ).

\section{After 2 weeks:}

The mean protrusive movement for group I was $10.1 \pm$ $1.9 \mathrm{~mm}$ and the mean protrusive movement for group II was $9.6+1.5 \mathrm{~mm}$. The decrease in protrusive movement from preoperative to 2 weeks postoperative was found to be statistically significant in group I (p-value $=0.01$ ) and also in group II (p-value $=0.04$ ).

\section{After 4 weeks:}

The mean protrusive movement for group I was $8.6 \pm$ $1.1 \mathrm{~mm}$ and the mean protrusive movement for group II was $8.1+1.7 \mathrm{~mm}$. The decrease in protrusive movement from preoperative to 1 months postoperative was found to be statistically significant in group I $(\mathrm{p}$-value $=0.0009)$ and also in group II ( $\mathrm{p}$-value $=0.005$ )

\section{After 3 months:}

The mean protrusive movement for group I was $5.5 \pm$ $0.5 \mathrm{~mm}$ and the mean protrusive movement for group II was $5.6+1.2 \mathrm{~mm}$. The decrease in protrusive movement from preoperative to 3 months postoperative was found to be statistically significant in group I $(p-v a l u e=0.0001)$ and also in group II (p-value $=0.0001)$. Regarding protrusive movement, the difference between the two groups throughout the whole follow-up period was found to be statistically insignificant $(\mathrm{p}<0.05)$.

Table (2): Comparison between the two studied groups according to protrusive jaw movement

\begin{tabular}{|c|c|c|c|c|}
\hline & Pre-operative & 2 weeks & 1 month & 3 months \\
\hline $\begin{array}{c}\text { Group I } \\
\text { Min. - Max. } \\
\text { Mean } \pm \text { SD } \\
\text { Median }\end{array}$ & $12.3 \pm 2$ & $9.6 \pm 1.4$ & $8.6 \pm 1.1$ & $5.5 \pm 0.5$ \\
& 1.3 & 1 & 0.8 & 0.5 \\
\hline p1 & & $0.01^{*}$ & $0.0009^{*}$ & $0.0001^{*}$ \\
\hline $\begin{array}{c}\text { Group II } \\
\text { Min. - Max. } \\
\text { Mean } \pm \text { SD } \\
\text { Median }\end{array}$ & $12.1 \pm 1.4$ & $10.1 \pm 1.9$ & $8.1 \pm 1.7$ & $5.6 \pm 1.2$ \\
\hline $\mathbf{p}_{\mathbf{1}}$ & 12 & 9 & 7 & 5 \\
\hline $\mathbf{p}_{2}$ & 0.43 & 0.31 & 0.34 & 0.38 \\
\hline
\end{tabular}

$\mathrm{p}_{1}$ : $\mathrm{p}$ value for paired t-test between preoperative and each other periods p2: $\mathrm{p}$ value for paired t-test between the two groups of the same period *: Statistically significant at $\mathrm{p} \leq 0.05$ 


\section{DISCUSSION}

TMJ dislocation is a distressing condition associated with pain and disability to close the mouth which causes interference with daily activities. Chronic TMJ dislocation is mainly due to laxity of the supporting ligaments, muscle hyperactivity or spasm, weakness of TMJ capsule, articular eminence abnormality in size, shape or projection, poor development of the articular fossa and some disorders such as Ehler- Danlous syndrome which is due to defect in collagen metabolism (20).

This study was conducted on twenty patients complaining from clinical signs and symptoms of chronic TMJ dislocation. In the present study, group (I) underwent injection of $3 \mathrm{ml}$ of autologous blood ,While group (II) underwent injection of $3 \mathrm{ml}$ of PRP under local anesthesia. In this study, there was a significant decrease in mandibular range of motion in both groups postoperatively in comparison to preoperative measurements on the other hand there was no statistical significant difference between the two groups

Autologous blood injection is a simple procedure and is considered a non-invasive technique for the treatment of recurrent TMJ dislocation (23). In this study, autologous blood injection was applied to 10 patients. At the end of the 3 months follow-up period, the symptoms had improved in all patients.

Machon et al. (9) treated 25 patients diagnosed with chronic recurrent TMJ dislocation with bilateral injections of autologous blood into the upper joint space and bilaterally around the TMJ capsules. Twenty patients had a successful outcome and required no further treatment after the 1-year follow-up. Also, Aurora et al (24) successfully treated a 22-year-old with recurrent bilateral TMJ dislocation using autologous blood injection in the upper joint space and around the capsule; after the 1-year followup, there was no recurrence.

Candirli et al (25) used magnetic resonance imaging (MRI) to evaluate the effects of autologous blood injection in 14 patients who were imaged preoperatively and at 1 month postoperatively. All of the patients showed no TMJ dislocation 1 month after the injection.

Bayoumi et al (26), applied autologous blood injection to 15 patients of chronic bilateral dislocation. At the end of the 1-year follow-up period, the symptoms had improved in 12 patients and CBCT imaging that was done at 1 year postoperative exhibited no osseous changes for all of the participants in the study (26).

In this study, it was found that there was excessive mandibular range of motion of mandibular movement of patients with chronic TMJ dislocation. These findings are in agreement with Bayoumi et al (26) , who found that the MIO of patient with TMJ dislocation was excessive with mean measurement of $41.3 \mathrm{~mm}$.

The cause of excessive mandibular range of motion of patients with chronic TMJ dislocation is explained by Hasson and Nahlieli (23) who said that when the mandible is forced to be opened in case of laughing, yawning, vomiting, and forced mandibular opening during dental treatment or some medical procedures, such as endoscopies and direct bronchoscopies excessive looseness of the mandibular and capsular ligaments occurs. This looseness may cause hypermobility of the mandible, which may lead to dislocation of the condyle (23).
Patients with condyle dislocation will seek treatment because this is a condition that interferes with their daily activities. Most of these patients will restrain their mandibular opening when laughing, yawning, or biting because they are afraid of getting their mandible too far anteriorly to the eminence, which could cause the mandible to lock while open (dislocation) (23) .

Autologous blood injection is a simple procedure and is considered a non-invasive technique for the treatment of recurrent TMJ dislocation. The main purpose of autologous blood injection is the limitation of mandibular movements (26).

In our study, it was found that the mandibular range of motion decreased after autologous blood injection in TMJ and this is in agreement with Machon et al (9), Daif (27), Bayoumi et al (26) and Triantafillidou et al (28).

Our results are also in agreement with Machon et al (9) who found that the average of MIO was decreased from 40 $\mathrm{mm}$ to $35 \mathrm{~mm} 1$ year after autologous blood injection. Also, Daif (27) used autologous blood injection in patients with chronic TMJ dislocation and concluded that at the end of the follow-up period, 1 year the average decrease of the maximal mouth opening was $5.3 \pm 2.1 \mathrm{~mm}$.

In another study, Bayoumi et al (26) found that the MIO was decreased by $6.73 \mathrm{~mm}$, one year after injection of autologous blood in patients with chronic TMJ dislocation which coincide with our results.

Triantafillidou et al (28) in his study treated 25 patients with chronic TMJ dislocation by autologous blood injection and after 3 months follow up, significant reduction of MIO and TMJ sound was noted and this goes along with our study findings.

The proposed mechanism of action is that the blood injected to pericapsular region would result in formation of local bed of fibrous tissue and that blood injected into superior cavity of the joint combined with blood of pericapsular tissue might lead to formation of adhesion in the cavity leading to decrease of mandibular range of motion (23).

Regarding the second group of this study, the PRP injection of patients with chronic TMJ dislocation resulted in significant reduction in pain intensity this is in agreement with several studies that used PRP injection for patients suffering from TMJ disorder.

Hanci et al. (21) studied the intra-articular injection of PRP into the TMJ in patients with anterior disc displacement with reduction (study group) and compared the results with arthrocentesis (control group), and a statistically significant reduction in joint sound and an increase in mouth opening in the study group were found compared with the control group.

Cerza et al. (29) reported the use of platelet-rich plasma in the treatment of intra-articular cartilage injuries and knee inflammations; the treatment was reported to cause rapid reduction of pain and quick recovery of functional capability.

Pihut et al. (30) found significant reduction in pain intensity in patients with Temporomandibular dysfunction syndrome when $0.5 \mathrm{~mL}$ of plasma was injected into each TMJ in supplement to the basic prosthetic treatment.

Anitua et al. (31) showed that PRP, which contains growth factors, may have therapeutic effects in osteoarthritis by modulating synovial cell biology. They 
also reported an increased hyaluronic acid (HA) concentration and stabilized angiogenesis after platelet concentrate exposure.

Concerning mandibular range of motions, the results of this study showed significant reduction of mandibular range of motions after PRP injection in TMJ in patient with chronic dislocation. The comparison between the two studied groups showed no statistical significant difference in the reduction of mandibular range of motion. To the best of our knowledge, the review of literature is lacking studies on the effect of PRP injection on the mandibular range of motion of patients with chronic recurrent TMJ dislocation.

\section{CONCLUSION}

Both autologous blood and PRP are simple, low cost, and minimally invasive methods that allow a high concentrate of autologous growth factors in physiological proportions to be obtained from blood, which can be easily and safely injected directly into the TMJ space. Moreover, it is well known that the risk of allergy or infection is negligible due to the autologous nature of the platelet extract.

\section{REFRENCES}

1.Beek M, Koolstra J, Van Ruijven L, Van Eijden T. Threedimensional finite element analysis of the human temporomandibular joint disc. J Biomech. 2000;33:30716.

2.Pupo YM, Pantoja LLQ, Veiga FF, Stechman-Neto J, Zwir LF, Farago PV, et al. Diagnostic validity of clinical protocols to assess temporomandibular disk displacement disorders: a meta-analysis. Oral Surg, Oral Med, Oral Pathol. 2016.

3.Conti P, Miranda J, Araujo C. Relationship between systemic joint laxity, TMJ hypertranslation, and intraarticular disorders. Cran J. 2000;18:192-7.

4.Chou K, Chao S, Wang T, Hsu L. Recurrent spontaneously reduced unilateral temporomandibular joint dislocation masquerading as a transient ischemic attack. JACME. 2016;6:26-8.

5.Pradhan L, Jaisani MR, Sagtani A, Win A. Conservative management of chronic TMJ dislocation: an old technique revived. J. Oral Maxillofac. Surg. 2015;14:267-70.

6.Gulses A, Bayar GR, Aydintug YS, Sencimen M, Erdogan E, Agaoglu R. Histological evaluation of the changes in temporomandibular joint capsule and retrodiscal ligaments following autologous blood injection. J CranioMaxillofacial Surg. 2013;41:316-20.

7.Nitzan DW. Temporomandibular joint “open lock” versus condylar dislocation: signs and symptoms, imaging, treatment, and pathogenesis. J. Oral Maxillofac. Surg. 2002;60:506-11.

8.da Silva CG, Pachêco-Pereira C, Porporatti AL, Savi MG, Peres MA, Flores-Mir C, et al. Prevalence of clinical signs of intra-articular temporomandibular disorders in children and adolescents: A systematic review and meta-analysis. J Am Dent Assoc. 2016;147:10-8. e8.

9.Machon V, Abramowicz S, Paska J, Dolwick MF. Autologous blood injection for the treatment of chronic recurrent temporomandibular joint dislocation. J. Oral Maxillofac. Surg. 2009;67:114-9.

10.Dutt CS, Ramnani P, Thakur D, Pandit M. Botulinum toxin in the treatment of muscle specific Oro-facial pain: a literature review. J. Oral Maxillofac. Surg. 2015;14:171-5.
11.Schiffman E, Look J, Hodges J, Swift J, Decker K, Hathaway K, et al. Randomized effectiveness study of four therapeutic strategies for TMJ closed lock. J Dent Res. 2007;86:58-63.

12.Boudreaux R, Spire E. Plication of the capsular ligament of the temporomandibular joint: a surgical approach to recurrent dislocation or chronic subluxation. J Oral Surg. 1968;26:330.

13.Puelacher W, Waldhart E. Miniplate eminoplasty: a new surgical treatment for TMJ-dislocation. J CranioMaxillofacial Surg. 1993;21:176-8.

14.Gould J. Shortening of the temporalis tendon for hypermobility of the temporomandibular joint. J Oral Surg. 1978;36:781.

15. Miller GA, Murphy EJ. External pterygoid myotomy for recurrent mandibular dislocation: Review of the literature and report of a case. Oral Surg, Oral Med, Oral Pathol. 1976;42:705-16.

16.James P. The surgical treatment of mandibular joint disorders. Ann R Coll Surg Engl. 1971;49:310.3

17.Armijo-Olivo S, Pitance L, Singh V, Neto F, Thie N, Michelotti A. Effectiveness of manual therapy and therapeutic exercise for temporomandibular disorders: systematic review and meta-analysis. Phys. Ther. 2015;96:9-25.

18. Matsumoto A, Matsumoto K, Kakimoto N, Yura Y. Arthroscopic findings after autologous blood injection in the treatment of recurrent temporomandibular joint dislocation. Oral Surg, Oral Med, Oral Pathol. 2015;27:225-7.

19.Yoshioka N, Shimo T, Ibaragi S, Sasaki A. Autologous Blood Injection for the Treatment of Recurrent Temporomandibular Joint Dislocation. Acta Med Okayama. 2016;70:291-4.

20.Varedi P, Bohluli B. Autologous blood injection for treatment of chronic recurrent TMJ dislocation: is it successful? Is it safe enough? A systematic review. J. Oral Maxillofac. Surg. 2015;19:243-52.

21.Hanc1 M, Karamese M, Tosun Z, Aktan TM, Duman S, Savaci N. Intra-articular platelet-rich plasma injection for the treatment of temporomandibular disorders and a comparison with arthrocentesis. J Cranio-Maxillofacial Surg. 2015;43:162-6.

22.Kiliç SC,Güngörmüş $M$, Sümbüllü MA. Is arthrocentesis plus platelet-rich plasma superior to arthrocentesis alone in the treatment of temporomandibular joint osteoarthritis? A randomized clinical trial. J. Oral Maxillofac. Surg. 2015;73:1473-83.

23.Hasson O, Nahlieli O. Autologous blood injection for treatment of recurrent temporomandibular joint dislocation. Oral Surg, Oral Med, Oral Pathol. 2001;92:390-3.

24.Aurora J, Singh G, Kumar D, Kumar R, Singh K. Autologous injection for the treatment of recurrent temporomandibular joint dislocation-a case report. J Indian Dent Assoc. 2011;5:846-8.

25.Candirli C, Yüce S, Cavus UY, Akin K, Cakir B. Autologous blood injection to the temporomandibular joint: magnetic resonance imaging findings. Imaging Sci Dent. 2012;42:13-8.

26.Bayoumi A, Al-Sebaei M, Mohamed K, Al-Yamani A, Makrami A. Arthrocentesis followed by intra-articular autologous blood injection for the treatment of recurrent 
temporomandibular joint dislocation. J. Oral Maxillofac. Surg. 2014;43:1224-8.

27.Daif ET. Autologous blood injection as a new treatment modality for chronic recurrent temporomandibular joint dislocation. Oral Surg, Oral Med, Oral Pathol. 2010;109:31-6.

28.Triantafillidou K, Venetis G, Markos A. Short-term results of autologous blood injection for treatment of habitual TMJ luxation. J Cranio-Maxillofacial Surg. 2012;23:68992.

29.Cerza F, Carnì S, Carcangiu A, Di Vavo I, Schiavilla V, Pecora A, et al. Comparison between hyaluronic acid and platelet-rich plasma, intra-articular infiltration in the treatment of gonarthrosis. Am J Sports Med. 2012;40:2822-7.

30.Pihut M, Szuta M, Ferendiuk E, Zeńczak-Więckiewicz D.Evaluation of pain regression in patients with temporomandibular dysfunction treated by intra-articular platelet-rich plasma injections: a preliminary report. BioMed Res. Int. 2014;14:1-7.

31.Anitua E, Andia I, Ardanza B, Nurden P, Nurden AT. Autologous platelets as a source of proteins for healing and tissue regeneration. J Thromb Haemost. 2004;91:4-15.

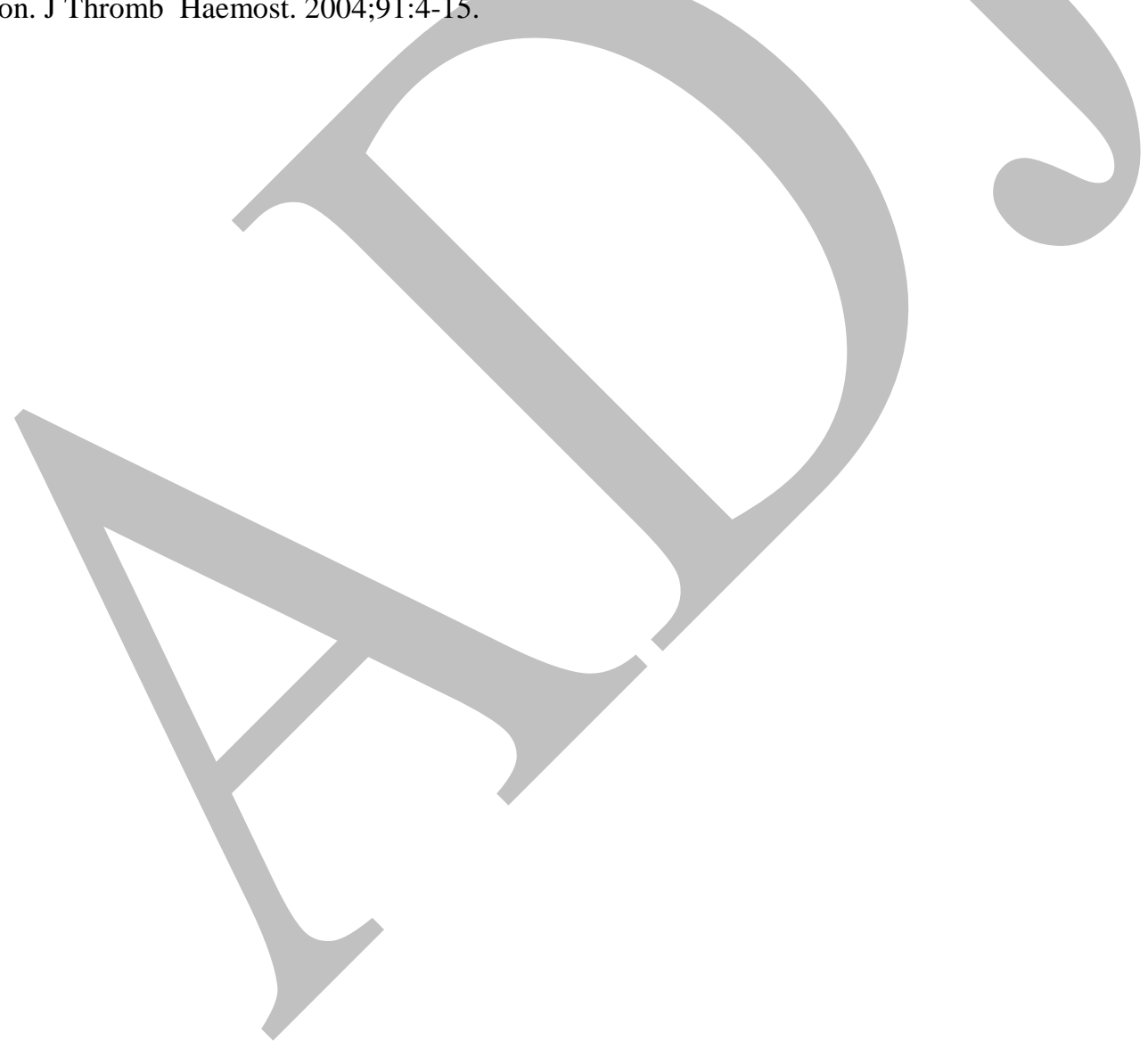

